\title{
Perioperative pain management in pediatric orthopaedic surgery
}

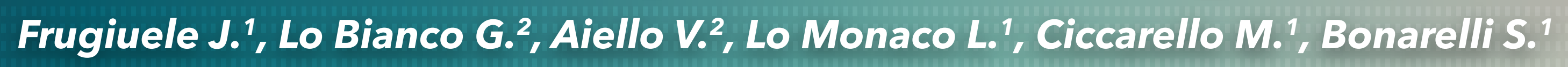

${ }_{1}^{1}$ Istituto Ortopedico Rizzoli Bologna, Dept of Anaesthesiology \& Intensive Care, Bologna, Italy

${ }^{2}$ Scuola di Specializzazione in Anestesia Università di Palermo, Dept of Anaesthesiology \& Intensive Care, Palermo, Italy

\section{Background and Goal of Study:}

Although regional anesthesia with neuraxial and peripheral blocks is a technique whose validity and safety has now been proven for some time, its use in pediatric surgery remains limited especially in Italy.

Our goal is to present results of a perioperative protocol in pediatric orthopedic surgery with patients aged between 5 and 14 years undergoing spinal anesthesia while under sedation and receiving a single-shot of US/ENS guided sciatic nerve block.

163 orthopedic surgery corrections of bilateral flat foot were performed between April 2012 and December 2015.

\section{Materials and methods:}

Patients were 89 male and 74 female, ASA I, from 5 to 14 years old. Signed consent forms were collected from both parents during the pre-hospitalisation visit. Patients were premedicated one hour before surgery with midazolam syrup $0.5 \mathrm{mg} / \mathrm{kg}$. We monitored standard vital signs and made IV sedation with ketamine $2 \mathrm{mg} / \mathrm{kg}$, atropine $0.01 \mathrm{mg} / \mathrm{kg}$ and midazolam $0.05 \mathrm{mg} / \mathrm{kg}$ iv.

Patients received spinal anesthesia with levobupivacaine $0.3 \mathrm{mg} / \mathrm{kg}$, by US identifing L4/L5 level.

We performed the ENS/US-guided single shot sciatic nerve block (subgluteal approach) by using levobupivacaine $1.5 \mathrm{mg} / \mathrm{kg}$ per side.

Surgery had an average duration of $15 \min \pm 10$ per side.

Once a plaster cast was fitted total OR time was $90 \mathrm{~min} \pm 15$.

Before discharging we evaluated vital signs and level of sedation using the Scale of sedation (SS) with Bromage 3 and facial pain scale (FPS)0.

Postoperative pain was managed by acetaminophen $15 \mathrm{mg} / \mathrm{kg}$ orally every 8 hours.

Rescue dose was set by Tramadol drops $1 \mathrm{mg} / \mathrm{kg}$ (lock-out 3 times per day) and recording FPS at rest and in motion every 6 hours until discharge.

\section{Results and discussion:}

Spinal anesthesia was successfully performed in all cases.

Only 1 child experienced a post-dural puncture headache lasting 4 days. It was treated with rehydration.

After surgery, the average FPS was 2 after 6 hours and 2.5 after 12 hours, while at discharge the average FPS was 1.1.

Five patients required pain treatment per os.

All patients were discharged on the third postoperative day.

\section{Conclusion:}

Regional anesthesia through central and peripheral blocks was found to be a safe technique, with complications comparable to those seen in adults.

The reduction of surgical stress and control of analgesia using advanced techniques influenced the outcome in terms of length of stay.

We found also strong evidence of postoperative analgesia and lack of significant perioperative complications
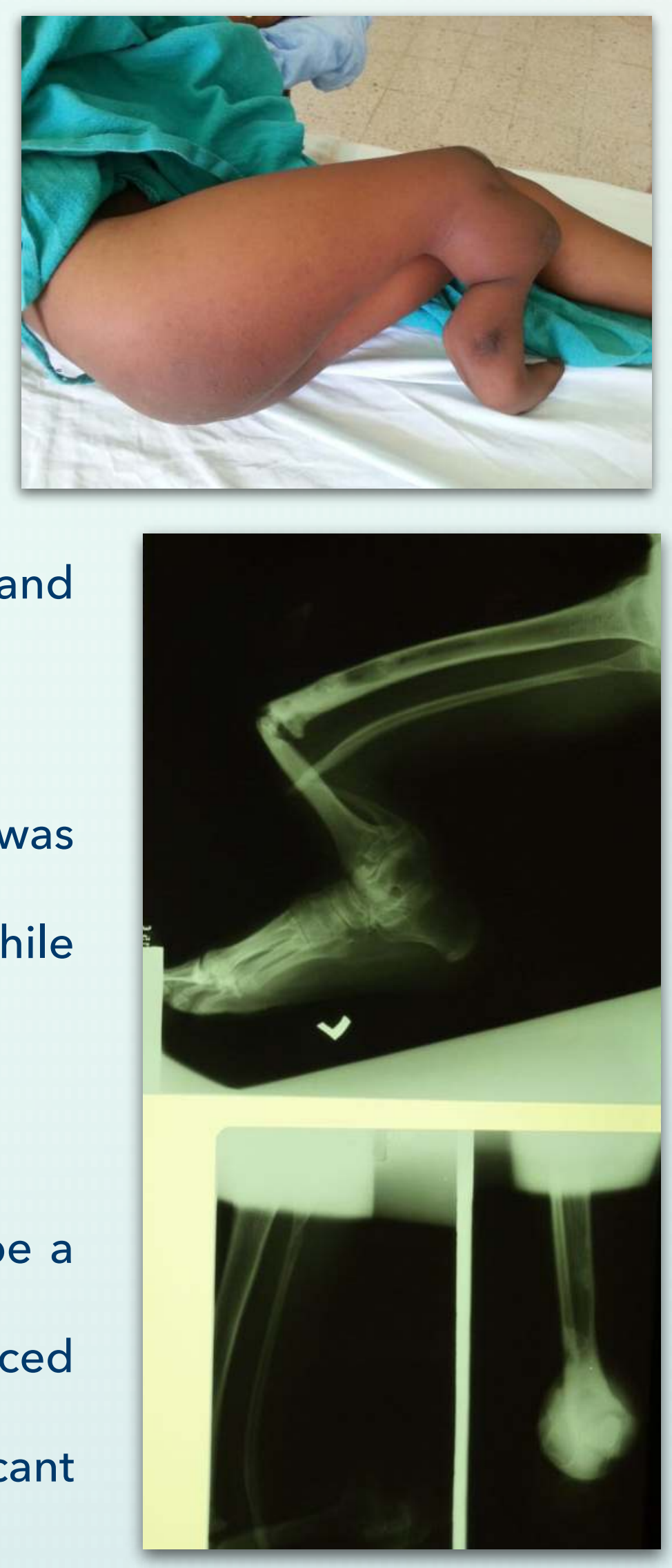